\title{
CONSERVACIÓN DE ORQUÍDEAS EN GUATEMALA: LA EXPERIENCIA DE UN COLEGIO
}

\author{
RAQUEL JIMÉNEZ DE PINTO
}

9 a. Calle 5-49, zona 10, Int.1, Ciudad de Guatemala, Guatemala

Desde 1994, los estudiantes del Colegio Ciudad Vieja de Ciudad de Guatemala trabajan en un proyecto de reforestación, conservación y rescate de orquídeas que crecen en un bosque de encino de aproximadamente 25 hectáreas (Barranco El Maestro) del Campus Central de la Universidad Francisco Marroquín (Ciudad de Guatemala, Guatemala). El proyecto forma parte esencial del programa de estudios de la institución y se realiza los sábados y domingos.

El primer año se realizó un censo de los árboles que crecen en el área, estableciendo que hay aproximadamente 6400 árboles, en su mayoría encino, ciprés, níspero, pino, aguacate, guayabo, jacaranda y casuarina. Inicialmente se determinó la presencia de trece especies de orquídeas, entre las que destacan por su abundancia: Barkeria skinneri Bateman ex Lindl., Comparettia falcata Poepp. \& Endl., Dichaea sp., Encyclia panthera (Rchb.f.) Schltr., Epidendrum sp., Macroclinium bicolor (Lind1.) Dodson, Maxillaria variabilis Batem. ex Lindl., y Ponera striata Lindl. En los últimos años el inventario se ha enriquecido con la localización de las siguientes especies: Cattleya aurantiaca (Batem. ex Lindl.) P.N. Don, Catlleya skinneri Batem., Dichromanthus cinnabarinus (La Llave \& Lex.) Garay, Epidendrum difforme Jacq., Oncidium (Trichocentrum) cavendishianum Bateman, Pleurothallis ghiesbreghtiana A. Rich. \& Galeotti y Ponthieva racemosa (Walt.) Mohr.

Los estudiantes también han realizado giras a otras zonas de la Ciudad de Guatemala con el propósito de rescatar orquídeas caídas de los árboles. En este proceso han resembrado miles de plantas de 56 especies de orquídeas. También han podido encontrar poblaciones naturales de 28 especies terrestres, incluyendo las siguientes: Bletia campanulata La Llave \& Lex.,
Bletia purpurea (Lam.) DC, Cranichis hieroglyphica Ames \& Correll, Cyclopogon elatus (Sw.) Schltr., Cyclopogon prasophyllum (Rchb.f.) Schltr., Spiranthes (Aulosepalum) pyramidalis Lindl., Goodyera striata Rchb.f., Govenia dressleriana E.W. Greenw., Govenia sp, Sobralia macrantha Lindl., Habenaria alata Hook., Habenaria limosa (Lind1.) Hemsl., Habenaria quinqueseta (Michx.) Sw., Habenaria sp., Malaxis parthonii Morren y Sarcologlottis cerina (Lindl.) P.N. Don.

El proceso de inventario de plantas ha continuado en los últimos años incrementando la lista de especies. También los estudiantes del colegio han complementado el proyecto con inventarios de fauna del Barranco El Maestro. Los resultados les han permitido comprender la interrelación entre plantas y animales, principalmente lo referente a polinización y dispersión de frutos y semillas. Además, el contacto con profesionales en áreas de las ciencias naturales ha mejorado notablemente el proceso de formación académica de los estudiantes y ayudado a su orientación vocacional.

Los resultados de este proyecto han sido ampliamente divulgados en ferias, exposiciones internacionales, seminarios y conferencias en Guatemala, Canadá, los Estados Unidos, El Salvador y Costa Rica. Los mismos estudiantes han elaborado material impreso y varios audiovisuales de excelente calidad. Este proyecto ha recibido muchos premios por parte de la Asociación Salvadoreña de Orquideología, la Asociación Costarricense de Orquideología, la Asociación Guatemalteca de Orquideología y reconocimientos por parte de la American Orchid Society. Especial mención merece el segundo lugar obtenido por el puesto educativo que los estudiantes presentaron en la Exposición Mundial de Orquídeas realizada en Vancouver, Canadá. Recientemente su 
trabajo fue objeto de un reportaje en la revista Selecciones del Readers Digest.

En los últimos dos años otros colegios de Ciudad de Guatemala han adoptado este modelo de conservación y enseñanza del Colegio Ciudad Vieja y están iniciando proyectos similares en otras zonas del área metropolitana de Guatemala, con lo cual se espera aumentar la conciencia pública y contribuir significativamente con la conservación de las orquídeas nativas de la Ciudad de Guatemala.

Raquel Jiménez de Pinto es profesora, directora y fundadora del Colegio Ciudad Vieja de Guatemala. Está graduada en pedagogía, sicología y tiene estudios de posgrado en ciencias sociales. Fue presidenta de la Asociación Guatemalteca de Orquideología. 